\title{
Van der Waals Emulsions: Emulsions Stabilized by Surface- Inactive, Hydrophilic Particles via van der Waals Attraction
}

\author{
Paula Marina Facal, Chongling Cheng, Rossen Sedev, Antonio Stocco, Bernard P. Binks and Dayang \\ Wang*
}

\begin{abstract}
Surface-inactive, highly hydrophilic particles are utilized to effectively and reversibly stabilize oil-in-water emulsions. This is a result of attractive van der Waals forces between particles and oil droplets in water, which are sufficient to trap the particles in close proximity to oil-water interfaces when the repulsive forces between particles and oil droplets are suppressed. The emulsifying efficiency of the highly hydrophilic particles is determined by the van der Waals attraction between the particle monolayer shells and the oil droplets enclosed therein and is inversely proportional to the particle size, while their stabilizing efficiency is determined by the van der Waals attraction between single particles and oil droplets which is proportional to the particle size. This differentiation in mechanism between emulsification and stabilization will significantly advance our knowledge of emulsions, thus enabling better control and design of emulsion-based technologies in practice.
\end{abstract}

Myriads of natural and industrial products are in the form of emulsions, in which fine oil droplets are well dispersed in water or vice versa by mechanical means with the aid of stabilizing agents. ${ }^{[1,2]}$ In the context of nanotechnology, nowadays emulsions are increasingly appreciated as soft, flexible, versatile toolboxes to produce smart, hierarchically structured materials. ${ }^{[3-6]}$ In almost all cases, emulsions need to be stabilized by surface-active agents: surfactants, amphiphilic polymers, solid particles or their combinations. By "surface-active", it is meant that the agents are able not only to adsorb onto oil-water interfaces but also straddle the interface by projecting themselves partially into oil and partially into water. ${ }^{[1,7]}$ Up to date, the partial partitioning of surface-active agents at oil-water interfaces is universally accepted as the key to emulsion stabilization, which enables them to self-assemble into topologically ordered films and thereby stabilize the dispersed droplets by, for instance, electrical double layer (EDL) repulsion against coalescence.$^{[1,7]}$ Counter-intuitively, here we show effective stabilization of oil-in-water (o/w) emulsions with surface-inactive agents-solid particles with exceedingly hydrophilic surfaces hardly wetted by oil at oil-water

[^] Dr. Paula Marina Facal, A/Prof. Rossen Sedev

Future Industries Institute, University of South Australia, Mawson Lakes, SA 5095 (Australia)

Chongling Cheng and Prof. Dayang Wang

State Key Laboratory for Inorganic Synthesis and Preparative Chemistry, College of Chemistry, Jilin University, Changchun 130012 (China)

Email: wangdayang@jlu.edu.cn

Dr. Antonio Stocco

Laboratoire Charles Coulomb (L2C), UMR 5221 CNRS-University of Montpellier, F-34095 (France)

Prof. Bernard P. Binks

School of Mathematics and Physical Sciences, University of Hull, Hull, HU6 7RX (UK)

Supporting information for this article is given via a link at the end of the document. interfaces. Our results reveal that provided the EDL repulsion between the particles and oil droplets is suppressed, the van der Waals (VW) attraction between them is sufficiently strong to confine the particles in close proximity to oil droplet surfaces to form densely packed shells which efficaciously envelop the oil droplets for emulsion stabilization. In this new process, intriguingly, the emulsifying power of the highly hydrophilic particles is governed preferentially by the VW attraction between the densely packed particle shell as a whole and the oil droplets enclosed therein, while their stabilizing power is governed by the VW attraction between individual particles and oil droplets. To highlight the contribution of VW attraction, here the present o/w emulsions are referred to as "VW emulsions".
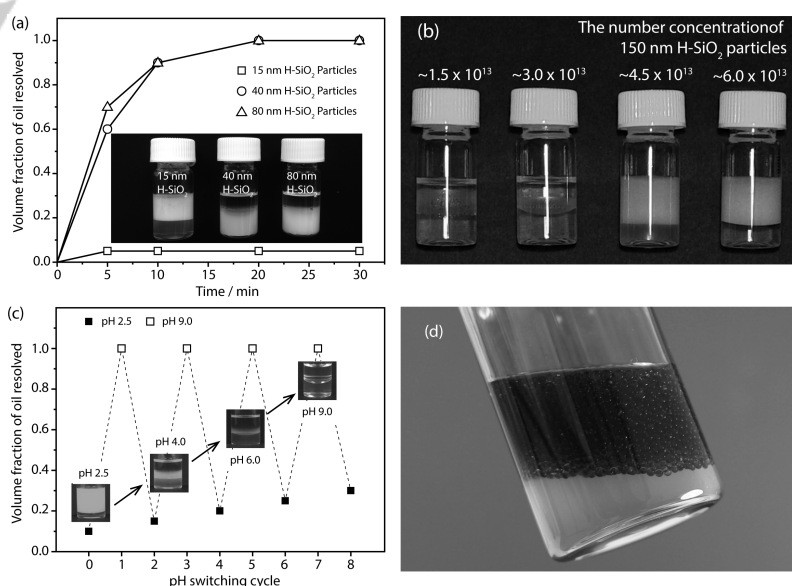

Figure 1. (a) Formation of VW emulsions by homogenization of toluene and aqueous dispersions of $15 \mathrm{~nm} \mathrm{H}-\mathrm{SiO}_{2}$ particles at $\mathrm{pH} 2.5$, evidenced by milky upper phase (emulsion) and transparent lower phase (water) in the glass vial and negligible recovery of free oil after emulsification. No emulsions can be stabilized by 40 and $80 \mathrm{~nm} \mathrm{H}-\mathrm{SiO}_{2}$ particles, evidenced by well-separated oil and water phases in the glass vials and fast and complete recovery of free oil phase emulsification. The concentrations of 15,40 , and $80 \mathrm{~nm} \mathrm{H}-\mathrm{SiO}_{2}$ particles are $5.4 \times 10^{15}, 8.8 \times 10^{14}$, and $9.8 \times 10^{13}$ particles $/ \mathrm{mL}$. (b) Dependence of the $\mathrm{VW}$ emulsion stabilization on the concentration of $150 \mathrm{~nm} \mathrm{H}^{2}-\mathrm{SiO}_{2}$ particles in the aqueous phase at $\mathrm{pH} 2.5$. The particle concentrations are marked on the photos. (c) Reversible stabilization of $\mathrm{VW}$ emulsions in response to $\mathrm{pH}$. The inset indicates that the VW emulsions, stabilized by $15 \mathrm{~nm} \mathrm{H}$-SiO2 particle at a concentration of $5.4 \times 10^{15}$ particles $/ \mathrm{mL}$ at $\mathrm{pH} 2.5$, significantly destabilize upon increasing the $\mathrm{pH}$ to 4.0 and completely collapse at $\mathrm{pH}$ above 6.0 .The $\mathrm{pH}$ adjustment is executed by dropwise addition of $0.1 \mathrm{M} \mathrm{NaOH}$ or $0.1 \mathrm{M} \mathrm{HCl}$ into the emulsions, followed by incubation for ca. $12 \mathrm{~h}$ under ambient condition. (d) Stabilization of VW emulsions (the upper phase in the photo) by citratestabilized gold particles of diameters of $16 \mathrm{~nm}$ at $\mathrm{pH} 2.5$. The oil phase is toluene in all experiments

According to thermodynamics, the depth $(\Delta \mathrm{E})$ of the potential well that traps solid particles at an oil-water interface with tension $\left(\gamma_{o w}\right)$ is correlated with the contact angle $(\theta)$ of the particles at the

This is the peer reviewed version of the following article: P. F. Marina, C. Cheng, R. Sedev, A. Stocco, B. P. Binks, D. Wang, Angew. Chem. Int. Ed. 2018, 57, 9510., which has been published in final form at https://doi.org/10.1002/anie.201805410. This article may be used for non-commercial purposes in accordance with Wiley Terms and Conditions for Use of Self-Archived Versions. 
interface and their radius $(r)$, which is symmetrically expressed with respect to $\theta=90^{\circ}$ as: ${ }^{[8]}$

$$
\Delta E=\pi \gamma_{o w} r^{2}(1 \pm \cos \theta)^{2}
$$

The magnitude of $\Delta \mathrm{E}$ can easily be $>10^{3} \mathrm{kT}$ ( $\mathrm{k}$ is the Boltzmann constant and $\mathrm{T}$ is the absolute temperature) for solid particles with $\theta$ in the range of $20^{\circ}-90^{\circ}$, even when $r \approx 5 \mathrm{~nm}$. Once adsorbed at the oil-water interface, therefore, the particles with such intermediate surface wettability are almost irreversibly trapped resulting in the outstanding long-term stability of particlestabilized emulsions, known as Pickering emulsions. When $\theta<$ $20^{\circ}, \Delta \mathrm{E}$ quickly diminishes so highly hydrophilic particles can hardly be held at the interface to stabilize a Pickering emulsion. In stark contrast, here we demonstrate that highly hydrophilic particles with $\theta \approx 0^{\circ}$ can effectively and reversibly stabilize an o/w emulsion. The VW emulsions are stabilized by small particles more effectively than by large ones, while the interchange of differently sized particles readily occurs between the surfaces of the dispersed oil droplets and the continuous water phase. These unique features make the VW emulsions reminiscent of surfactant-stabilized ones. In contrast, Pickering emulsions are more effectively stabilized by large particles rather than by small ones and thus the preferential displacement of small particles by large particles on dispersed droplets was observed when $r<2.5$ $\mathrm{nm}$ according to equation $1^{[9]}$.

Here uniform, spherical, silica particles with diameters of either 15, 40, 80, 150, and $200 \mathrm{~nm}$ were synthesized in water (Figure S1 and Table S1) and thoroughly cleaned to ensure the particle surfaces as well as the particle dispersions were completely free of the organic additives (Figure S2). As-prepared naked, hydrophilic silica particles, denoted as $\mathrm{H}_{-} \mathrm{SiO}_{2}$, were redispersed in water $(\mathrm{pH}$ 5.5). When as-prepared particle dispersions were mixed with apolar organic solvents such as toluene and hexadecane, no stable emulsions were formed even under intense homogenization (Figure S3). When the $\mathrm{pH}$ of the as-prepared particle dispersions is adjusted to 2.5 , intriguingly, 15 $\mathrm{nm} \mathrm{H}-\mathrm{SiO}_{2}$ particles can effectively stabilize an o/w emulsion simply by hand shaking but larger particles cannot even by intense homogenization (Figures $1 \mathrm{a}$ and S4). Nevertheless, $\mathrm{H}$ $\mathrm{SiO}_{2}$ particles with diameter $>15 \mathrm{~nm}$ become effective in emulsion stabilization at $\mathrm{pH} 2.5$ when the particle concentration in water is noticeably increased. For instance, $150 \mathrm{~nm} \mathrm{H}-\mathrm{SiO}_{2}$ particles can effectively stabilize o/w emulsions at $\mathrm{pH} 2.5$ after the as-prepared particle dispersions are concentrated by $>5$ times (Figure $2 b$ ). The resulting o/w emulsions destabilize with an increase in $\mathrm{pH}$ and are completely broken into well-separated oil and water phases at $\mathrm{pH}>6.7$, while they are effectively re-stabilized upon lowering the $\mathrm{pH}$ back to 2.5 (Figure 1c). Since the isoelectric points of both $\mathrm{H}-\mathrm{SiO}_{2}$ particles and oil droplets in water are close to $\mathrm{pH} 2.5^{[10]}$ (Figure S5), the $\mathrm{pH}$-response of the resulting o/w emulsion stabilization reflects the presence of $\mathrm{pH}$-dependent EDL repulsion between the $\mathrm{H}-\mathrm{SiO}_{2}$ particles and oil droplets. Similar to $15 \mathrm{~nm} \mathrm{H}-\mathrm{SiO}_{2}$ particles, citrate-stabilized gold particles with diameter of $16 \mathrm{~nm}$ can also effectively stabilize oil droplets in water at $\mathrm{pH} 2.5$ (Figure 1d), at which the EDL repulsion between the citrate-laden surfaces of gold particles and oil droplet surfaces is minimized, implying the irrelevance of the surface chemical nature of highly hydrophilic particles in stabilizing o/w emulsions.
In order to evaluate the partitioning of $\mathrm{H}_{-} \mathrm{SiO}_{2}$ particles at the oil-water interface at $\mathrm{pH} 2.5$, the refractive index profile across the planar interface was accessed by means of ellipsometry according to our previous study ${ }^{[11]}$. Figure 2 a reveals significant changes in ellipsometric scans at the toluene-water interface after pure water is replaced by aqueous dispersions of $200 \mathrm{~nm}$ silica particles with partially hydrophobized surfaces. These particles straddle the toluene-water interface with a contact angle through water of $125 \pm 2^{\circ}$ and a surface coverage of $0.7 \pm 0.2$ based on a particle monolayer model at the interface ${ }^{[11]}$. In contrast, no noticeable difference in ellipsometric scans is observed in the presence or absence of $200 \mathrm{~nm} \mathrm{H}-\mathrm{SiO}_{2}$ particles at $\mathrm{pH}$ 2.5. This points to the absence of particles at the interface or to such weak adsorption which cannot be detected by ellipsometry. This is in good agreement with excellent resistance of naked silica surfaces against attachment of oil droplets in water at pH 2.5 (Figure S6) and little surface activity of as-prepared $\mathrm{H}-\mathrm{SiO}_{2}$ particles at $\mathrm{pH} 2.5$ (Figure S7). Thus, we can conclude that $\mathrm{H}-\mathrm{SiO}_{2}$ particles are hardly (if at all) wetted by the oil phase at the toluene-water interface. Figure $2 \mathrm{~b}$ shows that the $\mathrm{o} / \mathrm{w}$ emulsions stabilized by $200 \mathrm{~nm} \mathrm{H}-\mathrm{SiO}_{2}$ particles at $\mathrm{pH} 2.5$ undergo complete destabilization after being frozen to $-18{ }^{\circ} \mathrm{C}$ and thawed to room temperature, again implying non-wetting of the particles by the oil phase at the interface. In contrast, Pickering emulsions stabilized by $200 \mathrm{~nm}$ silica particles with partially hydrophobized surfaces are sufficiently stable against the freeze-thaw action, since $\Delta \mathrm{E}$ arising from the partial wetting of $200 \mathrm{~nm}$ particles $(r=100 \mathrm{~nm})$ with reasonably hydrophilic surfaces $\left(\theta=30^{\circ}\right)$ at the oil-water interface is over $7000 \mathrm{kT}$ (Figure 2b).
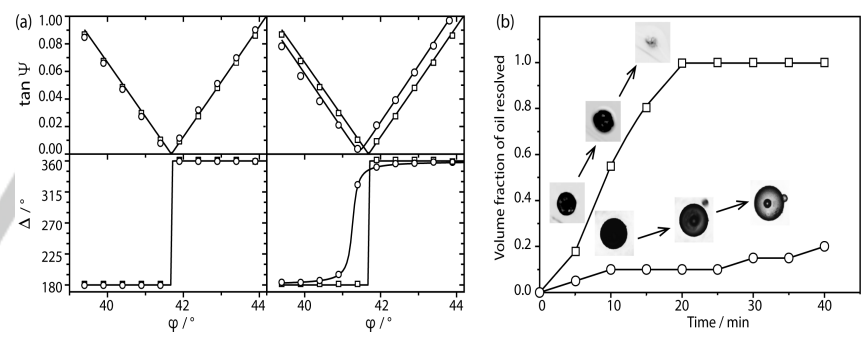

Figure 2. (a) Ellipsometric scans at the oil-water interface before (open squares) and after (open circles) addition of $200 \mathrm{~nm} \mathrm{H}-\mathrm{SiO}_{2}$ particles (left panels) and 200 $\mathrm{nm} \mathrm{SiO} 2$ particles with partially hydrophobized surfaces (right panels), $\mathrm{pH}=2.5$. Lines are the fits to the data of amplitude $\tan \Psi$ (upper panels) and phase shift (lower panels) as a function of the incident angle. The particle concentration is ca. $1 \times 10^{10}$ particles $/ \mathrm{mL}$. (b) Destabilization of VW emulsions, stabilized by 200 $\mathrm{nm} \mathrm{H}$-SiO2 particles at $\mathrm{pH} 2.5$, by freeze-thaw action; the free oil phase is completely recovered after $20 \mathrm{~min}$ thawing at room temperature. The particle concentration is ca. $5 \times 10^{12}$ particles $/ \mathrm{mL}$. In contrast, Pickering emulsions stabilized by $200 \mathrm{~nm}$ partially hydrophobized $\mathrm{SiO}_{2}$ particles are stable against freeze-thaw action. The oil phase is toluene in all experiments.

To effectively stabilize o/w emulsions, obviously, not only minimal EDL repulsion but also sufficient strong attraction between particles and dispersed oil droplets are necessitated in order to confine the $\mathrm{H}-\mathrm{SiO}_{2}$ particles in close proximity to the surfaces of the oil droplets in water at $\mathrm{pH} 2.5$. Hydrophobic attraction can be ruled out taking into account non-wetting of $\mathrm{H}$ - 
$\mathrm{SiO}_{2}$ particles by the oil phase at the oil-water interface (Figure 2a) and excellent oil-repellence of naked silica surfaces in water at $\mathrm{pH} 2.5$ (Figure S6). The $\mathrm{VW}$ attraction is therefore the only interaction trapping the $\mathrm{H}-\mathrm{SiO}_{2}$ particles in close proximity to the surfaces of dispersed oil droplets to form densely packed shells that envelop the oil droplets. To test our hypothesis, apolar organic solvents with different refractive indices were utilized as the oil phase (Table S2). Figure 3 shows effective stabilization of o/w emulsions by $15 \mathrm{~nm} \mathrm{H}-\mathrm{SiO}_{2}$ particles at $\mathrm{pH} 2.5$ when the refractive index of the oil phase, e.g. hexadecane, toluene and xylene, is significantly larger than that of water to create positive Hamaker constant (Table S2) and thus VW attraction between particles and dispersed oil droplets in water. The stabilization efficiency noticeably declines as the refractive index decreases, e.g. octane and hexane. In contrast, no emulsion is formed when organic solvents of refractive index less than that of water are used, e.g. perfluorohexane, which creates VW repulsion between particles and oil droplets as a result of negative Hamaker constant (Table S2). ${ }^{[12]}$ The revealed refractive index effect supports the notion that the formation and stabilization of o/w emulsions is driven by the $\mathrm{VW}$ attraction between $\mathrm{H}-\mathrm{SiO}_{2}$ particles and oil droplets in water

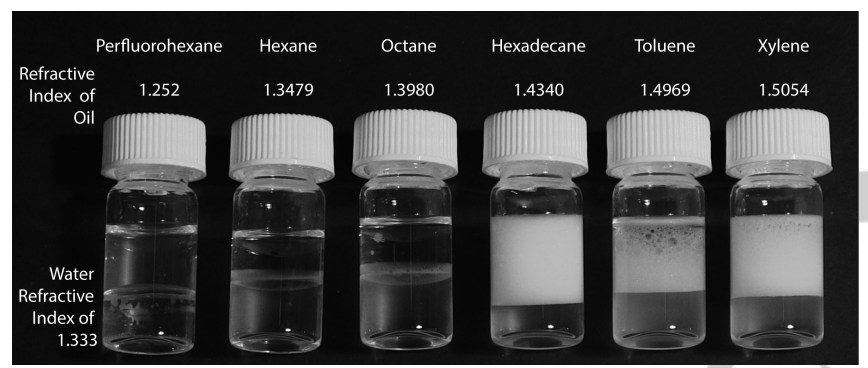

Figure 3. Dependence of the $\mathrm{VW}$ emulsion stabilization of $15 \mathrm{~nm} \mathrm{H}-\mathrm{SiO}_{2}$ particles at $\mathrm{pH} 2.5$ on the refractive index of the oil phase. The particle concentration is $5.4 \times 10^{15}$ particles $/ \mathrm{mL}$. The photos are taken in $6 \mathrm{~h}$ after homogenization of the oil and the particle dispersion.

To elucidate the formation and stabilization of VW emulsions, we start calculating the energy associated with VW attraction between $\mathrm{H}-\mathrm{SiO}_{2}$ particles and dispersed oil droplets in water. The oil droplets, stabilized by $15 \mathrm{~nm} \mathrm{H}-\mathrm{SiO}_{2}$ particles in water, are several hundred microns in size (Figure S4) and ca. 104 times larger than the particles, so the $\mathrm{VW}$ attraction between a $\mathrm{H}-\mathrm{SiO}_{2}$ particle with radius $r$ and a giant oil droplet through water $\left(W_{V W}^{p / o}\right)$ is simply given as follows: ${ }^{[12]}$

$$
W_{V W}^{p / o} \approx-A r / 6 x
$$

where $x$ is the surface-to-surface separation between the particle and the oil droplet and $A$ is the Hamaker constant (Table S2). Equation 2 indicates a linear increase of $W_{V W}^{p / o}$ with $r$ (Figure 4), which cannot account for $15 \mathrm{~nm} \mathrm{H}-\mathrm{SiO}_{2}$ particles being superior to large ones in emulsion formation and stabilization (Figure 1a) or the particle concentration effect (Figure 1b). For effective stabilization of o/w emulsions, oil droplets must be armored by densely packed particle shells. Hence we consider the VW energy of a number of particles $(\mathrm{N})$ participating in the stabilization of a single oil droplet and estimate the VW attraction between a monolayer shell of densely packed particles and an oil droplet with radius $\mathrm{R}$ enclosed therein $\left(W_{V W}^{p S / o}\right)$ as follows:

$$
W_{V W}^{p S / o} \approx N W_{V W}^{p / o} \approx-A R^{2} / 6 x r
$$

where $N \approx R^{2} / r^{2}$ as $R \gg r$. In contrast to $W_{V W}^{p / o}, W_{V W}^{p S / o}$ increases with $1 / r$ and rises progressively to several $10^{7} k T$ when $r<$ $50 \mathrm{~nm}$ (Figure 4). This accounts well for the size-dependence of the emulsification effectiveness of $\mathrm{H}-\mathrm{SiO}_{2}$ particles (Figure 1a). It may also provide clues to the highly effective stabilization by surfactants $(r \approx 1 \mathrm{~nm})$ in spite of the very small value of $\Delta \mathrm{E}$ trapping them at an oil-water interface. For a given $r$, on the other hand, Equation 3 indicates that the $W_{v W}^{p s / o}$ increases with $\mathrm{N}$, which accounts for the particle concentration effect on emulsion formation (Figure 1b).

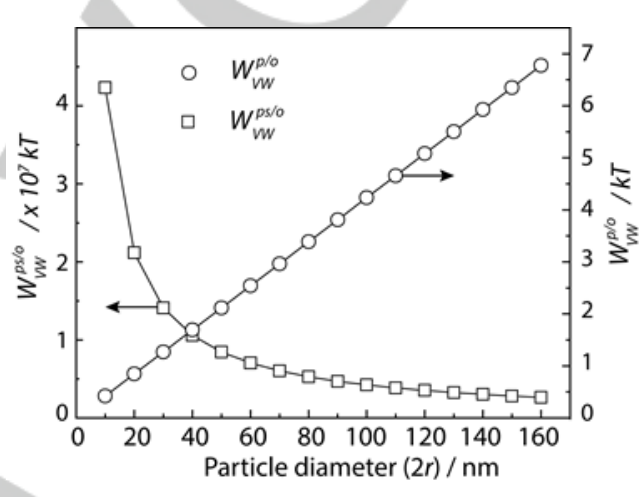

Figure 4. Variation of the values of $W_{V W}^{p / o}$ (open circles) and $W_{V W}^{p s / o}$ (open squares) as a function of particle diameters $(2 r)$ calculated based on Equation 2 and Equation 3, respectively. For simplicity of calculation, $x$ is set to $1.5 \mathrm{~nm}$ to achieve sufficiently large $W_{V W}^{p / o}$ and, at the same time, retain other possible repulsions such as hydration and osmotic interaction to avoid the near-surface contact between the particle and the oil droplet. Hexadecane is chosen as the oil phase for calculation.

To gain better understanding of the particle size effect on the formation of VW emulsions, apolar organic solvents were emulsified in aqueous dispersions of the binary mixtures of small and large $\mathrm{H}-\mathrm{SiO}_{2}$ particles at $\mathrm{pH} 2.5$, in which the numbers of the small and large particles were adjusted to be sufficient for either of them to effectively create the VW emulsion. Intriguingly, the resulting o/w emulsions are stabilized solely by the small particles, while the large particles remain exclusively in the continuous water phase (Figure 5a). This stresses that small $\mathrm{H}-\mathrm{SiO}_{2}$ particles are superior to large ones in emulsification. From a viewpoint of diffusion kinetics (Table S1), small particles reach the proximity to the oil-water interface faster than large one and thus may be the kinetic favorable for emulsification. This particle diffusivity effect on emulsification kinetics, however, may be largely smeared out under intense homogenization. Thus, it is plausible to postulate that the emulsifying power of $\mathrm{H}-\mathrm{SiO}_{2}$ particles is governed by $W_{V W}^{p S / o}$. To unravel the particle size effect on VW emulsion stabilization, the o/w emulsions, stabilized by small $\mathrm{H}-\mathrm{SiO}_{2}$ particles, were placed atop the aqueous dispersions of large $\mathrm{H}$ $\mathrm{SiO}_{2}$ particles after the free small particles were completely removed from the continuous water phase of the VW emulsions. The displacement of the small particles by the large ones 
becomes noticeable after 1 month and the complete displacement is achieved after 3 months, yielding VW emulsions stabilized solely by the larger particles (Figure $5 b$ ). By contrast, the substitution of small $\mathrm{H}-\mathrm{SiO}_{2}$ particles dispersed in the continuous water phase for large ones stabilizing the dispersed oil droplets is much less effective and becomes visible after 3 months (Figure 5c). These two sets of results stress that the large particles is superior to small ones in VW emulsion stabilization, thus implying that the stabilizing power of $\mathrm{H}-\mathrm{SiO}_{2}$ particles is governed by $W_{V W}^{p / o}$. It is worth noting that in as-prepared VW emulsions energetically favorable substitution of large $\mathrm{H}-\mathrm{SiO}_{2}$ particles for small ones on the dispersed oil droplets is surprisingly slow (ca. 3 months) taking into account that the particles remain non-wetted by the oil droplets and particle exchange occurs exclusively in the continuous water phase. This slow substitution may reflect the difficulty for small particles to escape from the densely packed particle shells enveloping the oil droplets, which requires the particles to overcome the VW attraction with the nearest neighboring particles. This inter-particle VW attraction may therefore offer an additional contribution to VW emulsion stability.
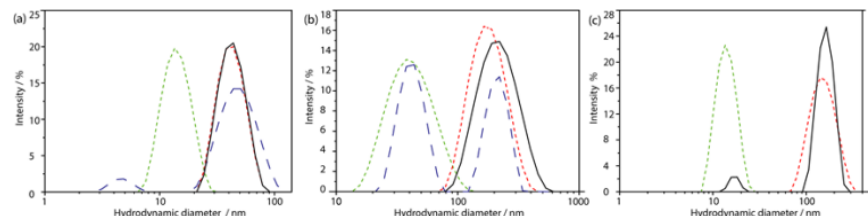

Figure 5. (a) Comparison of the intensity-averaged hydrodynamic diameter profiles of aqueous dispersions of $15 \mathrm{~nm}$ (green curve) and $40 \mathrm{~nm}$ (red curve) $\mathrm{H}-\mathrm{SiO}_{2}$ particles and mixtures of $15 \mathrm{~nm}$ and $40 \mathrm{~nm} \mathrm{H}-\mathrm{SiO}_{2}$ particles (blue curve) with that of the aqueous phase obtained after homogenization of the binary particle dispersion and toluene to form VW emulsions (black curve). (b) Substitution of large particles for small ones at toluene-water interfaces of VW emulsions via incubation of $40 \mathrm{~nm} \mathrm{H}-\mathrm{SiO}_{2}$ particle-stabilized emulsion in aqueous dispersion of $150 \mathrm{~nm} \mathrm{H}-\mathrm{SiO}_{2}$ particles for 1 (blue curve) and 3 months (black curve). (c) Substitution of small particles for large ones at the toluenewater interfaces of $\mathrm{VW}$ emulsions via incubation of $150 \mathrm{~nm} \mathrm{H}-\mathrm{SiO}_{2}$ particlestabilized emulsion in aqueous dispersion of $15 \mathrm{~nm} \mathrm{H}-\mathrm{SiO}_{2}$ particles for 3 months (black curve). Figures $b$ and $c$ show the intensity-averaged hydrodynamic diameter profiles of the aqueous phase obtained by destabilizing the VW emulsions via free-thaw action after particle exchange for a given time; the hydrodynamic diameter profiles of the corresponding small (green curve) and large particle dispersions (red curve) are shown for comparison. Note that in all experiments, the concentrations of small and large particles in water are adjusted to be sufficient for stabilization of VW emulsions by themselves alone.

Taken together, our results demonstrate that highly hydrophilic particles can effectively yet reversibly stabilize oil droplets in water as a result of the VW attraction between particles and oil droplets when the EDL repulsion between them is minimized. However, the resulting VW emulsions are vulnerable to external disturbance and subject to the dynamic exchange of different sized particles between the dispersed oil phase and the continuous water phase. These unique dynamic features make VW emulsions clearly distinct from Pickering emulsions, where solid particles are irreversibly trapped at oil-water interfaces via partial surface wetting, and largely reminiscent of surfactantstabilized emulsions. Our results underline that the formation of $\mathrm{VW}$ emulsions is controlled by $W_{V W}^{p S / o}$ but their stabilization is by $W_{V W}^{p / o}$. The revealed differentiation in mechanism between emulsification and stabilization should advance our knowledge of emulsions, especially those stabilized by binary surfactants or surfactants and solid particles, in which small surfactants appear more effective on emulsification ${ }^{[13]}$.

The present work underlines that the attractive forces between stabilizing agents and dispersed droplets, such as VW attraction and image charge attraction ${ }^{[14]}$, provide an important role in emulsion formation and stabilization, which must be sufficiently strong to ensure the stabilizing agents are effectively confined in close proximity to oil-water interfaces. It may offer a more generic model which enables us not only to articulate the pre-history of formation of emulsions before stabilizing agents adsorb to oil-water interfaces to form monolayer shells but also to rationalize the formation of unusual emulsions in the presence of ions such as hydroxide anions ${ }^{[10,15]}$. It may also unravel a subtle but important snapshot of how particles (as well surfactants) adsorb to oil-water interfaces from water, thus shedding light on many fundamental processes occurring at fluidic interfaces ${ }^{[16-18]}$. Taking into account the ubiquity of VW interactions, hopefully, our work will stimulate innovative designs of colloidal formulations in many disparate technical applications ranging from drug delivery for oral administration to bitumen emulsion for road construction.

\section{Acknowledgements}

Jilin University, RMIT University, and China Scholarship Council are acknowledged for the financial support of this work.

Keywords: Emulsion • Particles • Interfaces • Colloid • Interactions

[1] J. Israelachvili, Colloids Surf. A 1994, 91, 1-8.

[2] M. Chappat, Colloids Surf. A 1994, 91, 57-77.

[3] A. D. Dinsmore, Ming F. Hsu, M. G. Nikolaides, Manuel Marquez, A. R. Bausch, D. A. Weitz, Science 2002, 298, 1006-1009.

[4] V. N. Manoharan, M. T. Elsesser, D. J. Pine, Science, 2003, 301, 483-487.

[5] C. Burda, X. Chen, R. Narayanan, M. A. El-Sayed, Chem. Rev. 2005, 105, 1025-1102.

[6] M. P. Pileni, J. Phys. Chem. C 2007, 111, 9019-9038.

[7] H. T. Davis, Colloids Surf. A 1994, 91, 9-24.

[8] B.P. Binks, Langmuir 2017, 33, 6947-6963.

[9] B. Y. Lin, H. Skaff, T. Emrick, A. D. Dinsmore, T. P. Russell, Science 2003, 299, 226-229.

[10] J. K. Beattie, A. M. Djerdjev, Angew. Chemie Int. Ed. 2004, 43, 3568-3571.

[11] A. Stocco, G. Su, M. Nobili, M In, D. Wang, Soft Matter 2014, 10, 69997007.

[12] J. N. Israelachvili, Intermolecular and Surface Forces, Academic Press, New York, 3rd ed. 2009

[13] E. H. Lacassen-Reynders, Colloids Surf. A 1994, 91, 79-88.

[14] N. A. Elbers, J.E. S. van der Hoeven, D. A. Matthijs de Winter, C. T. W. M. Schneijdenberg, M. N. van der Linden, L. Filion, A. van Blaaderen, Soft Matter 2016, 12, 7265-7272.

[15] R. M. Pashley, J. Phys. Chem. C 2003, 107, 1714-1720.

[16] D. M. Kaz, R. McGorty, M. Mani, M. P. Brenner, V. N. Manoharan, Nature Mater. 2012, 11, 138-142.

[17] J. Samaniuk, J. Vermant, Soft Matter, 2014, 10, 7023-7033.

[18] G. Boniello, C. Blanc, D. Fedorenko, M. Medfai, N. Ben Mbarek, M. In, M. Gross, A. Stocco, M. Nobili, Nature Mater. 2015, 14, 908-912. 


\section{Entry for the Table of Contents}

\section{COMMUNICATION}

Surface-inactive, highly hydrophilic particles can effectively and reversibly stabilize oil-in-water emulsions thanks to van der Waals attraction between particles and oil droplets in water, thus referred to as van der Waals emulsion. In contrast to Pickering emulsions, the new emulsions are vulnerable to external disturbance and subject to the dynamic exchange of different sized particles between oil and water phases.

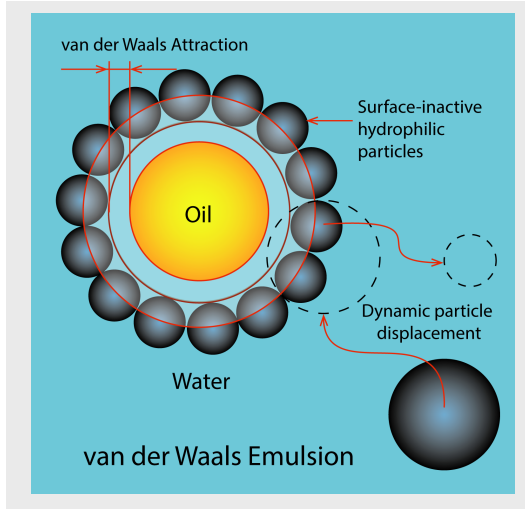

Paula M. Facal, Chongling Cheng, Rossen Sedev, Antonio Stocco, Bernard P. Binks, Dayang Wang*

\section{Page No. - Page No.}

Van der Waals Emulsions: Emulsions Stabilized by Surface-Inactive, Hydrophilic Particles via van der Waals Attraction 